3 Skakkebaek NE, Jorgensen N, Main KM et al: Is human fecundity declining? Int J Androl 2006; 29: 2-11.

4 Australian Bureau of Statistics (ABS): Births Australia 2006. Australian Capital Territory: Canberra, Australia, 2006. Catalogue number 3301.0. Available at: http://www.abs.gov.au/ AUSSTATS/abs@.nsf/web\%20pages/Citing\%20ABS\%20Sources\# Untitled\%20Section 8.

5 Sloter E, Nath J, Eskenazi B et al: Effects of male age on the frequencies of germinal and heritable chromosomal abnormalities in humans and rodents. Fertil Steril 2004; 81: 925-943.

\section{Reply to Herlihy and Halliday}

European Journal of Human Genetics (2008) 16, 1174; doi:10.1038/ejhg.2008.99; published online 21 May 2008

We thank the authors for their comments on our paper. They query as to why we do not mention paternal age as a possible explanation for the increase in prevalence of Klinefelters compared to XYY and XXX. We agree that it may be a partial explanation, but we do not believe that there is a substantial body of evidence of an association of paternal age with the birth of a child with Klinefelters. Of the five studies referenced in a recent meta-analysis, only one study found a significant positive association between paternal age and Klinefelters. ${ }^{1}$

The authors were correct in the assumption that we did not reference the study by Bojesen et $a l^{2}$ because it did not give the corresponding numbers of XYY and XXX diagnoses and the basis of our paper was to compare the prevalences of the three sex chromosome trisomies. The study by Bojesen et al covers the time period from 1970 to 2000 and they estimate a prenatal prevalence of 2.1 per 1000 (not specifying what proportion of diagnoses are from CVS or from amniocentesis), which compares to the data in our paper of 3.1 per 1000 observed in an amniocentesis series in women over age 35 from 1976-1981. It is difficult to directly compare these two figures as Klinefelters is associated with maternal age and has a fairly high fetal loss rate, so the gestational age at diagnosis is important.

Joan Morris ${ }^{*, 1}$, On behalf of the authors: Joan K Morris, Eva Alberman, Claire Scott, Patricia Jacobs

${ }^{1}$ Centre for Preventive Medicine Barts and the London, Queen Mary's School of Medicine and Dentistry, Wolfson Institute of Preventive Medicine, London, UK.

${ }^{*}$ Correspondence: $\mathrm{Dr} J$ Morris, Wolfson Institute of Preventive Medicine, Charterhouse Square, London, EC1M 6BQ United Kingdom. Tel: 207882 6274, Fax: 2078826270.

E-mail: j.k.morris@qmul.ac.uk

\section{References}

1 Sloter E, Nath J, Eskenazi B, Wyrobek AJ: Effects of male age on the frequencies of germinal and heritable chromosomal abnormalities in humans and rodents. Fertil Steril 2004; 81: 925-943.

2 Bojesen A, Juul S, Gravholt CH: Prenatal and postnatal prevalence of Klinefelter syndrome: a national registry study. J Clin Endocrinol Metabol 2003; 88: 622-626.

\section{Hypotheses in genome- wide association scans}

European Journal of Human Genetics (2008) 16, 1174-1175; doi:10.1038/ejhg.2008.97; published online 14 May 2008

Dear editor,

With great interest we have read your commentary on genome-wide association studies (GWAS), published in the January 2008 issue of this journal. ${ }^{1}$ In view of the recent interest in GWAS and the consequent impact on the side of both publishers and funding bodies, however, we think that some of the points raised in your buoyant contribution are worth further reflection.

Contrary to the view expressed in your commentary, GWAS do need an a priori hypothesis about the pathology of the disease under study, namely, that at least one causative genetic variant is statistically associated with at least one of the markers used. In fact, this is the conditio sine qua non of any GWAS. As good Popperians, we then hope for the GWAS to falsify the corresponding null hypothesis, that is, the complement of the above supposition. With linkage analysis (or 'positional cloning'), the situation is slightly different. There, physical proximity becomes the primary factor, rather than statistical correlation, so that the falsehood of the null hypothesis becomes a truism for virtually all marker panels currently used for genome-wide linkage analysis in humans.

In our view, understanding Popper's philosophy mainly as a strategy to optimize the unravelling of new truths is a gross misinterpretation. A cornerstone of his philosophy has been that scientific knowledge can only be achieved through falsification. If genetic epidemiologists feel that positive GWAS results still require 'replication', this is because they (rightly) regard the ensuing null hypotheses as falsifiable, and therefore 'scientific', claims in the sense of Popper.

Even with the impressive coverage provided by today's genotyping technologies, GWAS do not come anywhere near 'collecting all data required'. ${ }^{1}$ This is true, not only for rare genetic variation, but also for much of the common genetic variation in populations of non-European extraction.

Finally, 'thoroughly assessing [the] irrelevance' of putative genetic risk factors ${ }^{1}$ requires adequate data to be able to do so. Consideration of candidate genes becomes prohi- 